\title{
Front Matter: Volume 8621
}

, "Front Matter: Volume 8621," Proc. SPIE 8621, Optical Components and Materials X, 862101 (25 March 2013); doi: 10.1117/12.2022457

SPIE. Event: SPIE OPTO, 2013, San Francisco, California, United States 


\section{PROCEEDINGS OF SPIE}

\section{Optical Components and Materials $X$}

Michel J. F. Digonnet

Shibin Jiang

J. Christopher Dries

Editors

5-6 February 2013

San Francisco, California, United States

Sponsored and Published by

SPIE 
The papers included in this volume were part of the technical conference cited on the cover and title page. Papers were selected and subject to review by the editors and conference program committee. Some conference presentations may not be available for publication. The papers published in these proceedings reflect the work and thoughts of the authors and are published herein as submitted. The publisher is not responsible for the validity of the information or for any outcomes resulting from reliance thereon.

Please use the following format to cite material from this book:

Author(s), "Title of Paper," in Optical Components and Materials X, edited by Michel J. F. Digonnet, Shibin Jiang, J. Christopher Dries, Proceedings of SPIE Vol. 8621 (SPIE, Bellingham, WA, 2013) Article CID Number.

ISSN: 0277-786X

ISBN: 9780819493903

Published by

SPIE

P.O. Box 10, Bellingham, Washington 98227-0010 USA

Telephone +1 3606763290 (Pacific Time) · Fax +1 3606471445

SPIE.org

Copyright @ 2013, Society of Photo-Optical Instrumentation Engineers.

Copying of material in this book for internal or personal use, or for the internal or personal use of specific clients, beyond the fair use provisions granted by the U.S. Copyright Law is authorized by SPIE subject to payment of copying fees. The Transactional Reporting Service base fee for this volume is $\$ 18.00$ per article (or portion thereof), which should be paid directly to the Copyright Clearance Center (CCC), 222 Rosewood Drive, Danvers, MA 01923. Payment may also be made electronically through CCC Online at copyright.com. Other copying for republication, resale, advertising or promotion, or any form of systematic or multiple reproduction of any material in this book is prohibited except with permission in writing from the publisher. The CCC fee code is 0277-786X/13/\$18.00.

Printed in the United States of America.

Publication of record for individual papers is online in the SPIE Digital Library.

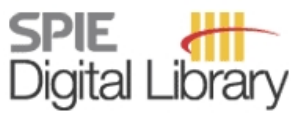

SPIEDigitalLibrary.org

Paper Numbering: Proceedings of SPIE follow an e-First publication model, with papers published first online and then in print and on CD-ROM. Papers are published as they are submitted and meet publication criteria. A unique, consistent, permanent citation identifier (CID) number is assigned to each article at the time of the first publication. Utilization of CIDs allows articles to be fully citable as soon as they are published online, and connects the same identifier to all online, print, and electronic versions of the publication. SPIE uses

a six-digit CID article numbering system in which:

- The first four digits correspond to the SPIE volume number.

- The last two digits indicate publication order within the volume using a Base 36 numbering

system employing both numerals and letters. These two-number sets start with 00, 01, 02, 03, 04 , $05,06,07,08,09,0 A, 0 B \ldots 0 Z$, followed by 10-1Z, 20-2Z, etc.

The CID Number appears on each page of the manuscript. The complete citation is used on the first page, and an abbreviated version on subsequent pages. Numbers in the index correspond to the last two digits of the six-digit CID Number. 


\title{
Contents
}

\author{
ix Conference Committee \\ xi Group IV photonics for the mid infrared (Plenary Paper) [8629-1] \\ R. Soref, The Univ. of Massachusetts at Boston (United States) \\ xxvii Light in a twist: optical angular momentum (Plenary Paper) [8637-2] \\ M. J. Padgett, Univ. of Glasgow (United Kingdom)
}

\section{SESSION $1 \quad$ NANO PHOTONICS}

862104 PbSe quantum dots grown in a high-index, low-melting-temperature glass for infrared laser applications [8621-3]

P. Kannan, A. Choudhary, B. Mills, V. M. Leonard, D. W. Hewak, X. Feng, D. P. Shepherd, Univ. of Southampton (United Kingdom)

862105 Bismuth nano-particle dispersed organic composite for optical components [8621-4] N. Kitamura, National Institute of Advanced Industrial Science and Technology (Japan); K. Takahashi, I. Mogi, Tohoku Univ. (Japan); K. Fukumi, National Institute of Advanced Industrial Science and Technology (Japan); S. Awaji, K. Watanabe, Tohoku Univ. (Japan)

\section{SESSION 2 NEW MATERIALS}

862106 SiPM for atmospheric Cherenkov telescopes [8621-5]

R. Mirzoyan, Max-Planck-Institut für Physik (Germany); E. Popova, National Research

Nuclear Univ. MEPhl (Russian Federation)

862107 Polish-like facet preparation via dicing for silica integrated optics [8621-6]

L. G. Carpenter, H. L. Rogers, C. Holmes, J. C. Gates, P. G. R. Smith, Univ. of Southampton (United Kingdom)

862108 Optical signal processing on photorefractive crystal substrate and bacteriorhodopsin thin film [8621-7]

M. Azimipour, R. Pashaie, Univ. of Wisconsin-Milwaukee (United States)

862109 High-sensitivity photoacoustic absorption spectroscopy of nonlinear optical materials [8621-8]

N. Waasem, Fraunhofer-Institut für Physikalische Messtechnik (Germany); K. Buse, Fraunhofer-Institut für Physikalische Messtechnik (Germany) and Albert-Ludwigs-Univ. Freiburg (Germany); F. Kühnemann, Fraunhofer-Institut für Physikalische Messtechnik (Germany)

$8621 \mathrm{OA} E r, \mathrm{Ce}: \mathrm{Y}_{3} \mathrm{Al}_{5} \mathrm{O}_{12}$ crystal properties under UV, VIS, and IR radiation exposure [8621-57] P. H. Muzhikyan, V. G. Babajanyan, R. B. Kostanyan, A. G. Petrosyan, Institute for Physical Research (Armenia) 
8621 OB Ultra-fast PDLC optical gate [8621-10]

O. Asmolova, Ampac Inc. (United States); R. Billmers, RL Associates Inc. (United States); T. Currant, Ampac Inc. (United States); Y. Garbovskiy, A. Glushchenko, Colorado Springs Univ. (United States); R. Lucente, P. Molchanov, Ampac Inc. (United States)

8621 OC An optical pulse width modulation generator based on the injection-locking property of single mode FP-LD [8621-11]

Q. H. Tran, B. Nakarmi, Y. H. Won, KAIST (Korea, Republic of)

8621 OD Multi-wavelength access gate for WDM-formatted words in optical RAM row architectures [8621-12]

D. Fitsios, T. Alexoudi, C. Vagionas, Ctr. for Research and Technology Hellas (Greece) and Aristotle Univ. of Thessaloniki (Greece); A. Miliou, Aristotle Univ. of Thessaloniki (Greece); G. T. Kanellos, Ctr. for Research and Technology Hellas (Greece); N. Pleros, Ctr. for Research and Technology Hellas (Greece) and Aristotle Univ. of Thessaloniki (Greece)

8621 OF Development of HgCdTe single-element APDs based detectors for low flux short wave infrared applications [8621-14]

K. Foubert, G. Lasfargues, L. Mathieu, S. Benahmed, G. Vojetta, J. Rothman, CEA-LETIMinatec (France); Q. Benoît à la Guillaume, V. Calvo, CEA, INAC/SP2M/SINAPS (France); J. Picot-Clemente, F. Le Mounier, F. Gibert, Lab. de Météorologie Dynamique, CNRS, Ecole Polytechnic (France)

\section{SESSION 4 FIBER SENSORS AND GRATINGS}

8621 0J Versatile chemical molecule sensing using multi-wavelength fiber laser based on intercore interference in twin-core photonic crystal fiber [8621-18] B. Kim, K. Naeem, J. Han, Y. Chung, Gwangju Institute of Science and Technology (Korea, Republic of)

$8621 \mathrm{OL} \quad$ Arc-induced long-period fiber gratings inscribed in asymmetric adiabatic tapers [8621-20] A. Martínez-Rios, D. Monzón-Hernández, G. Salceda-Delgado, Ctr. de Investigaciones en Óptica, A.C. (Mexico) 
8621 OM Tailored spectroscopic and optical properties in rare earth-activated glass-ceramics planar waveguides (Invited Paper) [8621-22]

D. Ristic, Istituto di Fotonica e Nanotecnologie (Italy); T. T. Van Tran, Univ. of Sciences (Viet Nam); B. Dieudonné, Univ. du Maine (France); C. Armellini, Istituto di Fotonica e Nanotecnologie (Italy); S. Berneschi, Museo Storica della Fisica e Ctr. Studi e Ricerche Enrico Fermi (Italy) and Istituto di Fisica Applicata Nello Carrara (Italy); A. Chiappini, A. Chiasera, S. Varas, A. Carpentiero, M. Mazzola, Istituto di Fotonica e Nanotecnologie (Italy); G. Nunzi Conti, S. Pelli, Istituto di Fisica Applicata Nello Carrara (Italy); G. Speranza, Fondazione Bruno Kessler (Italy) and Istituto di Fotonica e Nanotecnologie (Italy); P. Feron, ENSSAT-FOTON, CNRS, Univ. de Rennes 1 (France); C. Duverger Arfuso, Univ. du Maine (France); G. Cibiel, Ctr. National d'Études Spatiales (France); S. Turrell, LASIR, CNRS, CERLA, Univ. Lille 1 (France); K. Tran Ngoc, Hanoi Univ. of Science and Technology (Viet Nam); B. Boulard, Univ. du Maine (France); G. C. Righini, Museo Storica della Fisica e Ctr. Studi e Ricerche Enrico Fermi (Italy) and Istituto di Fisica Applicata Nello Carrara (Italy); M. Ferrari, Istituto di Fotonica e Nanotecnologie (Italy)

$8621 \mathrm{ON} \quad$ Improving $\mathrm{Ce}^{3+}$ doped scintillating materials for medical imaging applications [8621-23] S. Blahuta, Saint-Gobain Recherche (France) and LCMCP, Chimie-Paristech, UPMC, Collège de France (France); B. Viana, A. Bessière, D. Gourier, LCMCP, Chimie-Paristech, UPMC, Collège de France (France); V. Ouspenski, Saint-Gobain Recherche (France)

862100 White light emission characteristics of europium doped fluoride crystals [8621-24] B. R. Reddy, T. Colon, Alabama A\&M Univ. (United States)

$86210 Q \quad$ Study of lanthanum aluminum silicate glasses for passive and active optical fibers [8621-26]

K. Schuster, D. Litzkendorf, S. Grimm, J. Kobelke, A. Schwuchow, A. Ludwig, M. Leich, S. Jetschke, J. Dellith, Institut für Photonische Technologien e.V. (Germany); J.-L. Auguste, S. Leparmentier, G. Humbert, Xlim Institut de Recherche, CNRS (France); G. Werner, Fibotec Fiberoptics GmbH (Germany)

\section{SESSION 6 ACTIVE DEVICES AND BEAM CONTROL}

8621 OR Microresonator-based mid-IR devices (Invited Paper) [8621-27]

R. K. Jain, M. Hossein-Zadeh, The Univ. of New Mexico (United States)

8621 OS Microcavity-based cascaded Raman microlaser in air and in buffer [8621-28] M. V. Chistiakova, A. M. Armani, The Univ. of Southern California (United States)

$86210 U$ A noble refractive optical scanner with linear response [8621-30] Y. J. Mega, Z. Lai, C. A. DiMarzio, Northeastern Univ. (United States)

8621 OV Polygon mirror scanners in biomedical imaging: a review [8621-31] V.-F. Duma, Aurel Vlaicu Univ. of Arad (Romania); A. G. Podoleanu, Univ. of Kent (United Kingdom) 
8621 OW Photodarkening: investigation, mitigation and figure of merit (Invited Paper) [8621-32] H. Gebavi, S. Taccheo, Swansea Univ. (United Kingdom); L. LaBlonde, B. Cadier, T. Robin, iXFiber SAS (France); A. Monteville, O. Le Goffic, D. Landais, D. Méchin, D. Tregoat, PERFOS (France); D. Milanese, Politecnico di Torino (Italy); L. Leick, NKT Photonics A/S (Denmark); T. Durrant, Gooch and Housego (United Kingdom); T. Brand, DILAS Diodenlaser GmbH (Germany)

8621 OX Anomalous dispersive photonic bandgap fiber for ultra short pulse compression [8621-35] X. Yang, M. Y. Chen, Texas State Univ. San Marcos (United States)

$8621 \mathrm{OY}$ Environmental testing and laser transmission results for ruggedized high power IR fiber cables [8621-36]

L. Busse, U.S. Naval Research Lab. (United States); F. Kung, Univ. Research Foundation (United States); C. Florea, Sotera Defense Solutions, Inc. (United States); B. Shaw, U.S. Naval Research Lab. (United States); I. Aggarwal, Sotera Defense Solutions, Inc. (United States); J. Sanghera, U.S. Naval Research Lab. (United States)

$86210 Z$ Buckling conditions for a dual-coated optical fiber [8621-37]

E. Suhir, Bell Labs. (United States), Univ. of California, Santa Cruz (United States), Technische Univ. Wien (Austria), and ERS Co. (United States)

POSTER SESSION

862111 Supercontinuum generation from a multi-ring holes tellurite microstructured fiber pumped by a 2 micron high power mode-locked fiber laser [8621-33]

D. Deng, W. Gao, M. Liao, T. Suzuki, Y. Ohishi, Toyota Technological Institute (Japan)

862112 Analysis of soliton self-frequency shift in ZBLAN fiber as a broadband supercontinuum medium [8621-34]

X. Yan, Toyota Technological Institute (Japan) and Northeastern Univ. (China); M. Liao, T. Suzuki, Y. Ohishi, Toyota Technological Institute (Japan)

862113 Square-pulse operation in a ring cavity with a single-mode tellurite fiber [8621-38] W. Gao, M. Liao, H. Kawashima, T. Suzuki, Y. Ohishi, Toyota Technological Institute (Japan)

862114 All-solid tellurite-phosphate photonic bandgap fiber [8621-39] T. Cheng, M. Liao, H. T. Tong, W. Gao, Z. Duan, T. Suzuki, Y. Ohishi, Toyota Technological Institute (Japan)

862115 Photoluminescence and anti-deliquesce of cesium iodide and its sodium-doped films deposited by thermal evaporation at high deposition rates [8621-40] J.-C. Hsu, Y.-S. Chiang, Y.-S. Ma, Fu-Jen Catholic Univ. (Taiwan)

862116 Optical properties of cerium-codoped high power laser fibers [8621-41] S. Unger, A. Schwuchow, S. Jetschke, S. Grimm, A. Scheffel, J. Kirchhof, Institut für Photonische Technologien e.V. (Germany) 
862118 Fabrication of the reliable (14-18) $\times 1$ fiber laser power combiner by the novel double bundling method [8621-43]

B. H. Kim, Advanced Photonics Research Institute (Korea, Republic of); S.-J. Kim, Y. Yoon, Lighting Solution Technology Co. Ltd. (Korea, Republic of); S. Hann, Korea Photonics Technology Institute (Korea, Republic of)

862119 Classical and eclipse optical choppers [8621-44]

V.-F. Duma, Aurel Vlaicu Univ. of Arad (Romania)

$8621 \mathrm{lA}$ Upconversion and $1.5 \mu \mathrm{m}-1.6 \mu \mathrm{m}$ infrared emission studies of $\mathrm{Er}^{3+}$ doped in the low phonon-energy hosts $\mathrm{KPb}_{2} \mathrm{Cl}_{5}$ and $\mathrm{KPb}_{2} \mathrm{Br}_{5}$ via $1.5 \mu \mathrm{m}$ laser excitation [8621-45]

A. Bluiett, Elizabeth City State Univ. (United States); E. E. Brown, C. Hanley, U. Hömmerich, Hampton Univ. (United States); S. Trivedi, Brimrose Corp. of America (United States)

8621 1B Fluorescence quantum efficiency dependent on the concentration of $\mathbf{N d}^{3+}$ doped phosphate glass [8621-46]

A. A. Andrade, V. Pilla, Univ. Federal de Uberlândia (Brazil); S. A. Lourenço, Univ. Tecnológica Federal do Paraná (Brazil); A. C. A. Silva, N. O. Dantas, Univ. Federal de Uberlândia (Brazil)

8621 1C Elastomer-based tunable virtually imaged phased array for reconfigurable optical interconnects [8621-47]

P. Metz, C. Behnke, M. Gerken, Christian-Albrechts-Univ. zu Kiel (Germany); J. Adam, Christian-Albrechts-Univ. zu Kiel (Germany) and Univ. of California, Los Angeles (United States)

8621 ID Characterization of an SiPM dedicated at analytical, life science, and medical imaging [8621-48]

P. Bérard, M. Couture, F. Laforce, B. Fong, H. Dautet, Excelitas Technologies (Canada)

8621 IE A novel tellurite-phosphate glass for hybrid microstructured optical fibers [8621-49] Z. Duan, H. T. Tong, M. Liao, K. Asano, Toyota Technological Institute (Japan); M. Erwan, Univ. de Rennes (France); D. Sega, Y. Kano, K. Nagasaka, T. Suzuki, Y. Ohishi, Toyota Technological Institute (Japan)

$8621 \mathrm{lF}$ Mechanism of photonic crystal and waveguide effects in ZnO nanorods [8621-50] T. U. Kim, S. H. Kim, H. C. Ki, D. G. Kim, Korea Photonics Technology Institute (Korea, Republic of); M. G. Gang, Chonnam National Univ. (Korea, Republic of); H. J. Kim, Korea Photonics Technology Institute (Korea, Republic of); J. H. Kim, Chonnam National Univ. (Korea, Republic of)

8621 IG Optical characterization of Er-doped glasses for solar-pumped laser applications [8621-51] T. Suzuki, Y. Iwata, K. Nogata, Toyota Technological Institute (Japan); S. Mizuno, H. Ito, K. Hasegawa, Toyota Central R\&D Labs., Inc. (Japan); Y. Ohishi, Toyota Technological Institute (Japan) 
$86211 \mathrm{H} \quad$ UV-enhanced silicon avalanche photodiodes [8621-52]

R. A. Myers, R. Farrell, S. L. Riccardi, M. McClish, Radiation Monitoring Devices, Inc.

(United States)

$86211 \mathrm{~J} \quad$ Optical gain medium for plasmonic devices [8621-54]

V. A. G. Rivera, Univ. de São Paulo (Brazil) and Univ. Laval (Canada); Y. Ledemi, Univ. Laval (Canada); S. P. A. Osorio, F. A. Ferri, Univ. de São Paulo (Brazil); Y. Messaddeq, Univ. Laval

(Canada); L. A. O. Nunes, E. Marega Jr., Univ. de São Paulo (Brazil)

8621 1K High near-infrared emission intensity of $\mathrm{Er}^{3+}$-doped zirconium oxide films on a $\mathrm{Si}(100)$ substrate [8621-55]

V. A. G. Rivera, F. A. Ferri, Univ. de São Paulo (Brazil); J. L. Clabel H., Univ. Federal de São Carlos (Brazil); M. K. Kawamura, Univ. de São Paulo (Brazil); M. A. Pereira-da-Silva, Univ. de São Paulo (Brazil) and Ctr. Univ. Central Paulista, São Carlos (Brazil); L. A. O. Nunes, M. S. Li, E. Marega Jr., Univ. de São Paulo (Brazil)

$86211 \mathrm{~N} \quad$ A distinguishing method of different petrol by fiber-optic surface plasmon resonance sensor [8621-59]

D. Li, R. Zhu, P. Wu, K. Pang, J. Wu, Z. Zeng, K. Xu, Tianjin Univ. (China)

862110 Filamentation and supercontinuum generation in tellurite glass [8621-60]

M. Liao, W. Gao, T. Cheng, Z. Duan, X. Xue, H. Kawashima, T. Suzuki, Y. Ohishi, Toyota Technological Institute (Japan)

$86211 Q$ Coupled mode analysis for graded index multi-waveguide systems [8621-62]

K. C. Patra, Sambalpur Univ. (India); S. Srivastava, Univ. of Delhi (India); E. K. Sharma, Univ. of Delhi South Campus (India)

8621 is Yb :CALGO as material for high power ultrafast laser and focus on thermal conductivity variation [8621-64]

A. Jaffrès, Lab. de Chimie de la Matière Condensée de Paris, CNRS, Pierre-and-Marie-Curie Univ. (France); S. Ricaud, Lab. Charles Fabry, CNRS, Univ. Paris Sud (France); A. Suganuma, B. Viana, P. Loiseau, Lab. de Chimie de la Matière Condensée de Paris, CNRS, Pierre-andMarie-Curie Univ. (France); P. Georges, F. Druon, Lab. Charles Fabry, CNRS, Univ. Paris Sud (France)

8621 1T Optical properties of porous nano-composites of zinc (hydr)oxide with graphite oxide [8621-65]

S. M. Z. Islam, T. Gayen, M. Seredych, T. J. Bandosz, R. Alfano, The City College of New York (United States)

Author Index 


\section{Conference Committee}

Symposium Chair

David L. Andrews, University of East Anglia Norwich (United Kingdom)

Symposium Cochairs

Alexei L. Glebov, OptiGrate Corporation (United States)

Klaus P. Streubel, OSRAM GmbH (Germany)

Program Track Chair

James G. Grote, Air Force Research Laboratory (United States)

Conference Chairs

Michel J. F. Digonnet, Stanford University (United States)

Shibin Jiang, AdValue Photonics, Inc. (United States)

J. Christopher Dries, United Silicon Carbide, Inc. (United States)

Conference Program Committee

Jean-Luc Adam, Université de Rennes 1 (France)

Rolindes Balda, Universidad del País Vasco (Spain)

Robert P. Dahlgren, SETI Institute (United States)

Michael D. Gerhold, U.S. Army Research Office (United States)

Leonid B. Glebov, CREOL, The College of Optics and Photonics, University of Central Florida (United States)

Min Gu, Swinburne University of Technology (Australia)

Seppo K. Honkanen, University of Eastern Finland (Finland)

Lili Hu, Shanghai Institute of Optics and Fine Mechanics (China)

Jacques Lucas, Université de Rennes 1 (France)

Yasutake Ohishi, Toyota Technological Institute (Japan)

Aydogan Ozcan, University of California, Los Angeles (United States)

Giancarlo C. Righini, Istituto di Fisica Applicata Nello Carrara (Italy)

Feng Song, Nankai University (China)

Setsuhisa Tanabe, Kyoto University (Japan)

John M. Zavada, National Science Foundation (United States)

Session Chairs

1 Nano Photonics

John M. Zavada, National Science Foundation (United States) 
2 New Materials

Michel J. F. Digonnet, Stanford University (United States)

3 Optical Switch, Modulator, and Detector

Giancarlo C. Righini, Istituto di Fisica Applicata Nello Carrara (Italy)

4 Fiber Sensors and Gratings

Leonid Glebov, CREOL, The College of Optics and Photonics,

University of Central Florida (United States)

5 Rare-Earth Doped Materials

Rolindes Balda, Universidad del País Vasco (Spain)

6 Active Devices and Beam Control

Yasutake Ohishi, Toyota Technological Institute (Japan)

7 Novel Fibers

Shibin Jiang, AdValue Photonics, Inc. (United States) 